\title{
Gain smoothening filter in two-segment fiber-optical parametric amplifier.
}

\begin{abstract}
This paper demonstrates a method to remove the gain ripple profile specifically for a twosegment fiber optical parametric amplifier arising from the dispersion differences between the fiber gain medium and the standard single mode fiber attached to the optical components. This is achieved by using a simple design that incorporates a gain smoothening filter at the mid-stage of the amplifier. This simple yet practical method is useful when isolators are adopted for stimulated Brillouin scattering suppression but it comes with the expense of parametric gain.
\end{abstract}

Keyword: Parametric amplifier, gain smoothing filter 